\title{
Diophantine aspects of the Calkin-Wilf iteration
}

\author{
Jürgen Sander, Jörn Steuding, and Rasa Steuding
}

Jürgen Sander studierte Mathematik in Hannover und Boulder, Colorado. Seit 2009 ist er Professor für Algebra und Zahlentheorie an der Universität Hildesheim.

Rasa Steuding studierte und promovierte an den Universitäten in Šiauliai und Vilnius. Gegenwärtig arbeitet sie an der Hochschule RheinMain in Wiesbaden über Kryptographie.

Jörn Steuding promovierte an der Universität Hannover. Seit 2006 ist er Professor für Zahlentheorie an der Universität Würzburg.

Dedicated to Prof. Dr. K.-H. Indlekofer at the occasion of his 65th birthday

\section{The Calkin-Wilf matrix iteration}

The Calkin-Wilf tree is generated by the iteration

$$
\frac{a}{b} \mapsto \frac{a}{a+b}, \frac{a+b}{b},
$$

starting from the root $\frac{1}{1}$; we call $\frac{a}{a+b}$ the left child and $\frac{a+b}{b}$ the right child of $\frac{a}{b}$.

Obwohl die rationalen Zahlen bekanntlich abzählbar sind, ist eine „vernünftige“ systematische Aufzählung nicht offensichtlich. Eine übersichtliche Möglichkeit bietet der sogenannte Calkin-Wilf-Baum. Dieser Graph mit der Wurzel 1 listet sukzessive alle positiven rationalen Zahlen nach einer sehr einfachen Iterationsvorschrift auf. In dem nachfolgenden Beitrag betrachten die Autoren diese Iterationen aus dem Blickwinkel ganzzahliger $(2 \times 2)$-Matrizen mit Determinante 1 und können daraus Erkenntnisse für Calkin-Wilf-Bäume mit beliebigen reellen Wurzeln gewinnen. Damit können sie beispielsweise klären, welche Zahlen in einem Baum mit beliebiger reeller Wurzel auftreten. 
The first iterations lead to

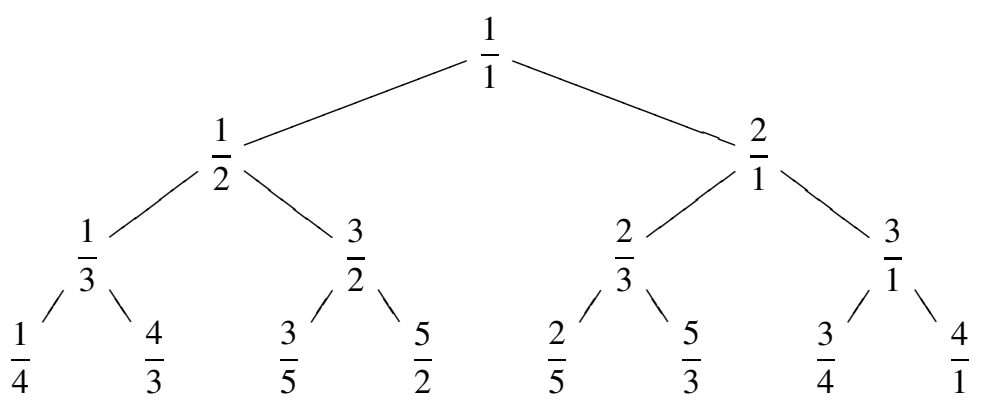

Calkin \& Wilf [5] have shown that this infinite tree contains any positive rational number once and only once, each of which represented as a reduced fraction. Reading the tree line by line, the Calkin-Wilf enumeration of $\mathbb{Q}^{+}$is starting as follows:

$$
\frac{1}{1}, \frac{1}{2}, \frac{2}{1}, \frac{1}{3}, \frac{3}{2}, \frac{2}{3}, \frac{3}{1}, \frac{1}{4}, \frac{4}{3}, \frac{3}{5}, \frac{5}{2}, \frac{2}{5}, \frac{5}{3}, \frac{3}{4}, \frac{4}{1}, \ldots
$$

This sequence satisfies the iteration

$$
x_{1}=1, \quad x_{n+1}=1 /\left(2\left[x_{n}\right]+1-x_{n}\right),
$$

where $[x]$ denotes the largest integer $\leq x$; this observation is due to Newman (cf. [13]), answering a question of Knuth, resp. Vandervelde \& Zagier (cf. [17]). The sequence of the denominators is called the Stern-Brocot sequence and was already investigated by Eisenstein [7] in 1850, Stern [18] in 1858, and Brocot [4] in 1860. The Calkin-Wilf enumeration of the positive rationals has many interesting features. For instance, it encodes the hyperbinary representations of all positive integers (see Calkin \& Wilf [5]), and it can be used as a model for the game Euclid (see Hofmann, Schuster \& Steuding [10]). A polynomial analogue was recently given by Dilcher \& Stolarsky [6]. There are quite many articles on this or related topics, e.g. the paper [11] of Lagarias \& Tresser on properties of the Farey tree. We apologize for the incomplete list of references and we are grateful for any information concerning publications with respect to this theme.

In this note we study new diophantine aspects of the Calkin-Wilf tree.

Consider the two $2 \times 2$ matrices

$$
U=\left(\begin{array}{ll}
1 & 0 \\
1 & 1
\end{array}\right) \quad \text { and } \quad T=\left(\begin{array}{ll}
1 & 1 \\
0 & 1
\end{array}\right)
$$

and their action as Möbius transforms on the upper half-plane

$$
U z=\frac{z}{z+1} \quad \text { and } \quad T z=z+1
$$

Specializing $z=\frac{a}{b}$ with positive integers $a, b$, we obtain the iteration which builds the Calkin-Wilf tree from the root $\frac{1}{1}$. Now denote by $I$ the unit matrix. Then we may read the 
Calkin-Wilf iteration as successive applications of the transforms $U$ and $T$ applied to $I$. The first generations of the corresponding tree are

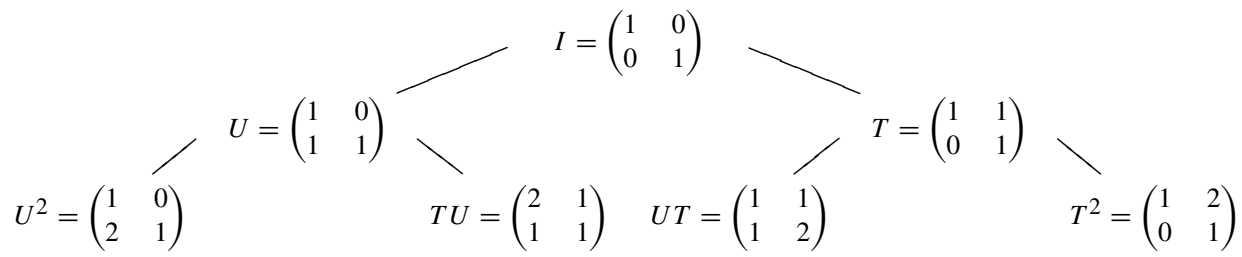

In the sequel we are concerned with the monoid $\Gamma_{\mathrm{CW}}$ consisting of all $\mathrm{PSL}_{2}(\mathbb{Z})$ matrices with non-negative integer entries and determinant 1 . Note that $\mathrm{PSL}_{2}(\mathbb{Z})=\mathrm{SL}_{2}(\mathbb{Z}) / \pm I$ is the modular group which is generated by the two matrices $T$ and $S=\left(\begin{array}{cc}0 & -1 \\ 1 & 0\end{array}\right)$. Our first aim is

Lemma 1. Any $M \in \Gamma_{\mathrm{CW}}$ has a unique representation

$$
M=U^{\ell_{n}} T^{r_{n}} \ldots U^{\ell_{2}} T^{r_{2}} U^{\ell_{1}} T^{r_{1}},
$$

where $\ell_{j}, r_{j} \in\{0,1\}$ such that $\ell_{j}+r_{j}=1$ for $j=1, \ldots, n$.

The lemma implies that $\Gamma_{\mathrm{CW}}$ is the injective image of the Calkin-Wilf iteration. The representation as a product of matrices $U^{\ell_{j}} T^{r_{j}}$ with $0 \leq \ell_{j}, r_{j} \leq 1$ and $\ell_{j}+r_{j}=1$ reflects the path from the root $I$ to the position of $M$ in the Calkin-Wilf tree; if $\ell_{j}=1$ the path turns to the left, otherwise $r_{j}=1$ and the path turns to the right in the $j$-th generation. The proof is more or less the same as the one of Calkin \& Wilf [5]. A different (tree-free) proof of the statement of the lemma was given by Alperin [2].

Proof. First of all we observe that

$$
U^{n}=\left(\begin{array}{ll}
1 & 0 \\
n & 1
\end{array}\right) \quad \text { and } \quad T^{n}=\left(\begin{array}{ll}
1 & n \\
0 & 1
\end{array}\right) .
$$

Since the entries of $U T$ and $T U$ are all positive, it follows that matrices $M$ obtained by the Calkin-Wilf iteration and having zero entries are of the form $M=U^{n}$ or $M=T^{n}$ for some $n \in \mathbb{N}_{0}$. Moreover, zero entries can only appear in non-diagonal positions. Now we prove the existence of the desired representation. For an arbitrary $M=\left(\begin{array}{ll}a & b \\ c & d\end{array}\right) \in \Gamma_{\mathrm{CW}}$ consider the matrices

and

$$
U^{-1} M=\left(\begin{array}{cc}
1 & 0 \\
-1 & 1
\end{array}\right)\left(\begin{array}{ll}
a & b \\
c & d
\end{array}\right)=\left(\begin{array}{cc}
a & b \\
c-a & d-b
\end{array}\right)
$$

$$
T^{-1} M=\left(\begin{array}{cc}
1 & -1 \\
0 & 1
\end{array}\right)\left(\begin{array}{ll}
a & b \\
c & d
\end{array}\right)=\left(\begin{array}{cc}
a-c & b-d \\
c & d
\end{array}\right),
$$

both having determinant 1 . Since $a d-b c=1$, exactly one of these matrices has nonnegative entries and belongs to $\Gamma_{\mathrm{CW}}$. We now assume that $\Gamma_{\mathrm{CW}}$ contains elements without 
the desired representation and choose $M=\left(\begin{array}{ll}a & b \\ c & d\end{array}\right)$ with this property and minimal trace $a+d$. Suppose that $U^{-1} M$ lies in $\Gamma_{\mathrm{CW}}$, having trace $a+d-b$. The minimality property of $M$ implies that $b=0$. Hence $U^{-1} M=\left(\begin{array}{cc}a & 0 \\ c-a & d\end{array}\right)$, and consequently $a=d=1$. Therefore, $U^{-1} M=\left(\begin{array}{cc}1 & 0 \\ c-1 & 1\end{array}\right)=U^{c-1}$, and we obtain the contradiction $M=U^{c}$. The other case $T^{-1} M \in \Gamma_{\mathrm{CW}}$ leads to the inconsistency $M=T^{b}$. It remains to prove that the representation is unique. Assume that $M$ has two representations, then so have both $U^{-1} M$ and $T^{-1} M$ too; again we get a contradiction by the minimality of the trace. The lemma is proved.

In the sequel we shall study the Calkin-Wilf iteration with respect to different initial values. This is not entirely new. For relations between continued fractions and the modular group we refer to Series [15] and Hockman [9]; indeed most of their methods apply to the CalkinWilf iteration, too.

\section{Continued fractions and equivalent numbers}

It is well-known that each real number $\alpha$ has a representation as a regular continued fraction

$$
\alpha=a_{0}+\frac{1}{a_{1}+\ddots \frac{1}{a_{m}+\ddots}}
$$

with $a_{0} \in \mathbb{Z}$ and $a_{m} \in \mathbb{N}$ for $m \in \mathbb{N}$. This representation is finite if and only if $\alpha$ is rational. In this case the representation is unique if the last partial quotient satisfies $a_{m} \geq 2$ provided $m \in \mathbb{N}$; if $\alpha$ is irrational, the continued fraction is infinite and the representation is unique. We shall use the standard notation $\alpha=\left[a_{0}, a_{1}, \ldots, a_{m}, \ldots\right]$. Continued fractions are of special interest in the theory of diophantine approximation. As Bird, Gibbons \& Lester [3] showed, the $n$-th generation of the rational Calkin-Wilf tree consists exactly of those rationals having a continued fraction expansion $\left[a_{0}, a_{1}, \ldots, a_{m}\right]$ for which the sum of the partial quotients $a_{0}+a_{1}+\ldots+a_{m}$ is equal to $n$ (actually, their reasoning is based on Graham, Knuth \& Patasnik [8] who gave such a description for the related Stern-Brocot tree).

Given a reduced fraction $\alpha$ in the rational Calkin-Wilf tree with continued fraction expansion

$$
\alpha=\left[a_{0}, a_{1}, \ldots, a_{m-2}, a_{m-1}, a_{m}\right],
$$

we associate the path

$$
\begin{aligned}
& \mathrm{L}^{a_{m}-1} \mathbf{R}^{a_{m-1}} \mathrm{~L}^{a_{m-2}} \ldots \mathrm{L}^{a_{1}} \mathbf{R}^{a_{0}} \quad \text { if } \quad m \text { is odd, and } \\
& \mathbf{R}^{a_{m}-1} \mathbf{L}^{a_{m-1}} \mathbf{R}^{a_{m-2}} \ldots \mathrm{L}^{a_{1}} \mathbf{R}^{a_{0}} \quad \text { if } m \text { is even; }
\end{aligned}
$$

note that $a_{m}-1 \geq 1$ for $m \in \mathbb{N}$. The notation $\mathrm{R}^{a}$ with $a \in \mathbb{N}_{0}$ means: $a$ steps to the right, whereas $\mathrm{L}^{b}$ with $b \in \mathbb{N}_{0}$ stands for $b$ steps to the left. Then, starting from the root $\frac{1}{1}$ and 
following this path from left to right, we end up with the element $\alpha$. This follows easily from the iteration with which the tree was built (this claim is essentially already contained in Lehmer [12] as observed by Reznick [14]) and so it can be generalized to other values for the root of the tree. However, we are interested in representations with respect to the matrix monoid $\Gamma_{\mathrm{Cw}}$. We observe that

$$
T\left[a_{0}, a_{1}, a_{2}, \ldots\right]=\left[a_{0}+1, a_{1}, a_{2}, \ldots\right] .
$$

For the mapping by $U$ we distinguish the cases $\alpha>1$ and $0<\alpha<1$; the case of $\alpha=1$ is covered by the rational Calkin-Wilf iteration. We find

$$
U\left[a_{0}, a_{1}, a_{2}, \ldots\right]= \begin{cases}{\left[0,1, a_{0}, a_{1}, a_{2}, \ldots\right]} & \text { if } a_{0}>0 \\ {\left[0, a_{1}+1, a_{2}, \ldots\right]} & \text { if } a_{0}=0 .\end{cases}
$$

Of course, the conditions on $a_{0}$ being positive or being equal to zero can be replaced by $\alpha>1$ or $0<\alpha<1$. We observe that in both cases, $T \alpha$ and $U \alpha$ have the same tail of the continued fraction expansion as $\alpha$.

Two real numbers $\alpha$ and $\beta$ are said to be equivalent if there exist integers $a, b, c, d$

$$
\beta=\frac{a \alpha+b}{c \alpha+d} \quad \text { with } \quad a d-b c= \pm 1 .
$$

Serret [16] proved that any two real numbers $\alpha$ and $\beta$ are equivalent if and only if their continued fraction expansions are identical from some index onwards (see also [19]). Consequently, equivalent numbers can be approximated by rationals with the same quality. More precisely, define the Markoff constant of a real number $\alpha$ by

$$
\lambda(\alpha)=\liminf _{q \rightarrow \infty} q\|q \alpha\|,
$$

where $\|x\|$ is the distance between $x$ and the nearest integer. Then $\lambda(\alpha)=\lambda(\beta)$ if $\alpha$ and $\beta$ are equivalent. It is easily seen that $\lambda(\alpha) \neq 0$ implies that the inequality

$$
\left|\alpha-\frac{p}{q}\right|<\frac{\lambda(\alpha)+\epsilon}{q^{2}}
$$

has infinitely many solutions in integers $p, q$ for any fixed positive $\epsilon$.

It is an easy exercise to show that equivalence of real numbers is indeed an equivalence relation and to deduce that any two rational numbers are equivalent. Thus, the Calkin-Wilf tree with root 1 is an enumeration of all positive numbers which are equivalent to 1 . If the root $\alpha$ of the Calkin-Wilf iteration is a quadratic irrational, we obtain an enumeration of all positive equivalent numbers to $\alpha$; however, the situation is different if the root is neither rational nor quadratic irrational.

Theorem 1. The Calkin-Wilf tree associated with a positive real number $\alpha$ contains any positive number $\beta$ of the form

$$
\beta=\frac{a \alpha+b}{c \alpha+d} \quad \text { with } \quad a, b, c, d \in \mathbb{N}_{0}, a d-b c=+1 ;
$$


all these numbers $\beta$ have the same tail in their continued fraction expansion as $\alpha$. The set of all these $\beta$ 's is an enumeration of all positive numbers equivalent to $\alpha$ if and only if $\alpha$ is rational or quadratic irrational; in the latter case there exist cycles.

First we illustrate the theorem by an example. Here we cleared the denominators from square roots and indicate, as usual, by a bar the period of a continued fraction expansion.

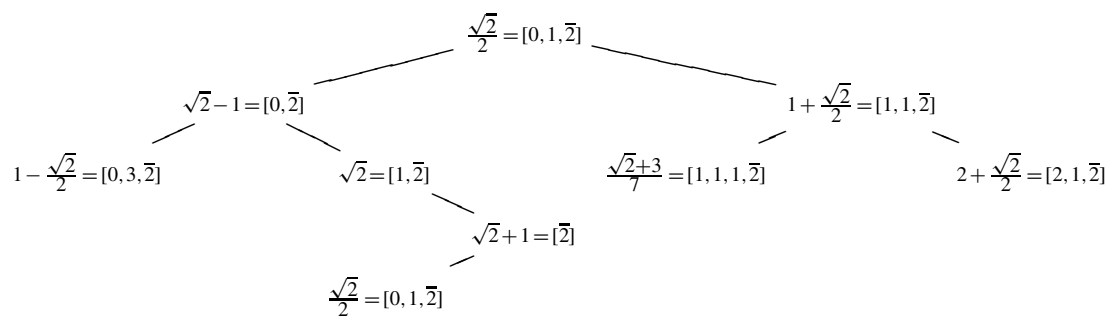

Indeed, we find that $z \mapsto U T T U z$ has the fixed point $z=\frac{\sqrt{2}}{2}$ (to check this note that $\left.U T T U z=\frac{3 z+2}{4 z+3}\right)$ and that $z \mapsto T U U T z$ has the fixed point $z=\sqrt{2}$.

Proof. First, we shall show that any positive equivalent number $\beta$ of the prescribed form is represented by the tree. Assume that $0<\alpha<1$ and that $\beta$ is equivalent to $\alpha$. Then, by Lemma 1, there exists a matrix $M \in \Gamma_{\mathrm{CW}}$ such that

$$
\beta=M \alpha=U^{\ell_{n}} T^{r_{n}} \ldots U^{\ell_{2}} T^{r_{2}} U^{\ell_{1}} T^{r_{1}} \alpha
$$

with $\ell_{j}, r_{j} \in\{0,1\}$. Hence, $\beta$ appears in the Calkin-Wilf tree with root $\alpha$.

Now we assume that $\alpha$ is neither rational nor quadratic irrational. Then there exist positive numbers $\beta$ which are equivalent to $\alpha$ but do not appear in the Calkin-Wilf tree associated with $\alpha$. To see that we consider

$$
\beta=\left(\begin{array}{cc}
-1 & n \\
0 & 1
\end{array}\right) \alpha=n-\alpha,
$$

which is positive if we take $n$ to be any integer greater than $\alpha$, and, by Serret's theorem, equivalent to $\alpha$; however, it is not of the prescribed form. Suppose it does, then $\beta=\left(\begin{array}{ll}a & b \\ c & d\end{array}\right) \alpha$ for $a, b, c, d \in \mathbb{N}_{0}$ and $a d-b c=1$. It follows that

$$
\left(\begin{array}{cc}
-1 & n \\
0 & 1
\end{array}\right)\left(\begin{array}{ll}
a & b \\
c & d
\end{array}\right) \alpha=\alpha
$$

and thus $\alpha$ would be the fixed point of the modular group, i.e., a root of a quadratic equation defined over $\mathbb{Z}$, a contradiction to our assumption.

It remains to consider fixed points. Given any $M=\left(\begin{array}{ll}a & b \\ c & d\end{array}\right) \in \mathrm{PSL}_{2}(\mathbb{Z})$, the fixed point equation $M z=z$ is either a linear or a quadratic equation in $z$. It is linear if and only if $c=0$ in which case a fixed point would be rational; however, thanks to the work of 
Calkin \& Wilf we know that there are no fixed points for any rational root $\alpha$. Otherwise, if $c \neq 0$, the equation is of degree two with integer coefficients, and so any fixed point is a quadratic irrational. It is well-known that an irrational number has an ultimately periodic continued fraction expansion if and only if it is quadratic irrational. Hence it follows that any element in a quadratic irrational Calkin-Wilf tree appears infinitely often. The theorem is proved.

To illustrate the last part of the proof note that

$$
\left(\begin{array}{cc}
0 & 1 \\
1 & -1
\end{array}\right) \sqrt{2}=\sqrt{2}+1=\left(\begin{array}{ll}
1 & 1 \\
0 & 1
\end{array}\right) \sqrt{2}
$$

this equation is false if we replace $\sqrt{2}$ by any number which is not a fixed point of $\left(\begin{array}{cc}-1 & 2 \\ 1 & -1\end{array}\right)$.

\section{Mean-value statistics}

Statistical properties of the rational Calkin-Wilf tree were (independently) investigated by Alkauskas \& Steuding [1], Reznick [14], and others. The set of all elements of the tree which are obtained by $n-1$ iterations is called the $n$-th generation and is denoted by $\mathrm{CW}^{(n)}=\left\{x_{j}^{(n)}\right\}_{j}$, where the $x_{j}^{(n)}$ are the elements ordered according to their appearance in the $n$-th line of the Calkin-Wilf tree. So $\mathbb{Q}^{+}=\bigcup_{n=1}^{\infty} \mathrm{CW}^{(n)}$. In $[1,14]$ the mean-value of the elements of the $n$-th generation of the rational Calkin-Wilf tree was computed. ${ }^{1}$ Actually, in [1] an exact formula for the sum $\Sigma(n)$ of all elements of the $n$-th generation of the Calkin-Wilf tree was obtained:

Theorem 2. For any $n \in \mathbb{N}$,

$$
\Sigma(n):=\sum_{j=1}^{2^{n-1}} x_{j}^{(n)}=3 \cdot 2^{n-2}-\frac{1}{2} .
$$

Since the number of elements of the $n$-th generation is $2^{n-1}$, the mean-value of $\mathrm{CW}^{(n)}$ is equal to $2^{1-n} \Sigma(n)$ which tends to $\frac{3}{2}$ as $n \rightarrow \infty$. For the sake of completeness we sketch the combinatorial proof from [1]:

Proof by induction on $n$. The statement of the theorem is correct for $n=1$ and $n=2$. Now suppose that $n \geq 3$. In order to prove the statement for $n$ we first observe a symmetry in the Calkin-Wilf tree with respect to its middle: for $n \geq 2$,

$$
x_{j}^{(n)}=\frac{a}{b} \quad \Longleftrightarrow \quad x_{2^{n-1}+1-j}^{(n)}=\frac{b}{a} ;
$$

this is easily proved by another induction on $n$ (and we leave its simple verification to the reader). Further, we note that $x_{j}^{(n)} \leq 1$ if and only if $j$ is odd; here equality holds if and only if $n=1$.

\footnotetext{
${ }^{1}$ Another approach is due to Wirsing who informed the second author by private communication.
} 
Now we start to evaluate $\Sigma(n)$. For this purpose we compute

$$
y_{j}^{(n)}:= \begin{cases}x_{j}^{(n)}+x_{2^{n-1}-j}^{(n)} & \text { for } j=1,2, \ldots, 2^{n-2}-1, \\ x_{j}^{(n)}+x_{2 j}^{(n)} & \text { for } j=2^{n-2}\end{cases}
$$

and add these values over $j=1,2, \ldots, 2^{n-2}$. Clearly, $\Sigma(n)=\sum_{j=1}^{2^{n-2}} y_{j}^{(n)}$.

First, assume that $j$ is odd. Then both, $x_{j}^{(n)}$ and $x_{2^{n-1}-j}^{(n)}$ are strictly less than 1 . In view of (1) the mothers of $x_{j}^{(n)}$ and $x_{2^{n-1}-j}^{(n)}$ are of the form $\frac{a}{b}$ and $\frac{b}{a}$, respectively. Hence,

$$
x_{j}^{(n)}+x_{2^{n-1}-j}^{(n)}=\frac{a}{a+b}+\frac{b}{a+b}
$$

and thus we find $y_{j}^{(n)}=1$ in this case.

Next, we consider the case that $j$ is even. Then both, $x_{j}^{(n)}$ and $x_{2^{n-1}-j}^{(n)}$ are strictly greater than 1 . If the mothers of $x_{j}^{(n)}$ and $x_{2^{n-1}-j}^{(n)}$ are of the form $\frac{a}{b}$ and $\frac{a^{\prime}}{b^{\prime}}$, respectively, then

$$
x_{j}^{(n)}=\frac{a+b}{b}=1+\frac{a}{b} \quad \text { and } \quad x_{2^{n-1}-j}^{(n)}=1+\frac{a^{\prime}}{b^{\prime}} .
$$

Hence, we find for their sum

$$
x_{j}^{(n)}+x_{2^{n-1}-j}^{(n)}=2+\frac{a}{b}+\frac{a^{\prime}}{b^{\prime}}
$$

and so $y_{j}^{(n)}=2+y_{k}^{(n-1)}$, where $y_{k}^{(n-1)}$ is either the sum of two elements $x_{k}^{(n-1)}$ and $x_{2^{n-2}-k}^{(n-1)}$ or the sum of $x_{2^{n-3}}^{(n-1)}$ and $x_{2^{n-2}}^{(n-1)}$.

It remains to combine both evaluations. Since both cases appear equally often, namely each $2^{n-3}$ times, we obtain the recurrence formula

$$
\Sigma(n)=\Sigma(n-1)+(1+2) \cdot 2^{n-3},
$$

being valid for $n \geq 3$. This implies the assertion of the theorem.

The finite mean-value $\frac{3}{2}$ has a simple explanation: in some sense, small values are taken in earlier generations than large values. For instance, in each generation $\mathrm{CW}^{(n)}$ takes as many values from the interval $(0,1)$ as from $(1, \infty)$. What happens if we start from another $\operatorname{root} \alpha$ ?

Theorem 3. For any $\alpha \in \mathbb{R}$ which is not a negative rational number or equal to zero, the mean-value of the Calkin-Wilf iteration with root $\alpha$ is equal to $\frac{3}{2}$.

We denote the $n$-th generation of the Calkin-Wilf tree with root $\alpha$ by $\operatorname{CW}^{(n)}(\alpha)$ and denote its elements by $x_{n}^{(j)}(\alpha)$, ordered in the same way as in the case $\alpha=1$. 
Proof. We observe that for all left children we have that $x_{j}^{(n)}(\alpha)-x_{j}^{(n)}$ tends to zero as $n \rightarrow \infty$. However, this is not sufficient to prove the theorem but an averaging over the whole generation will yield the proof. Put

$$
\Sigma_{n}:=\Sigma_{n}(\alpha):=\sum_{j=1}^{2^{n-1}}\left(x_{j}^{(n)}(\alpha)-x_{j}^{(n)}\right) .
$$

Since

$$
U z-U w=\frac{z-w}{(z+1)(w+1)}
$$

and

$$
T z-T w=z-w
$$

we have

$$
\begin{aligned}
\Sigma_{n} & =\sum_{j=1}^{2^{n-2}}\left(U x_{j}^{(n-1)}(\alpha)-U x_{j}^{(n-1)}+T x_{j}^{(n-1)}(\alpha)-T x_{j}^{(n-1)}\right) \\
& =\sum_{j=1}^{2^{n-2}}\left(x_{j}^{(n-1)}(\alpha)-x_{j}^{(n-1)}\right) \delta_{j}^{(n)}(\alpha)
\end{aligned}
$$

where

$$
\delta_{j}^{(n)}(\alpha):=1+\frac{1}{\left(x_{j}^{(n-1)}(\alpha)+1\right)\left(x_{j}^{(n-1)}+1\right)} .
$$

Next we want to show that

$$
x_{j}^{(n-1)}(\alpha) \geq U^{n-1} \alpha \gg \frac{1}{n}
$$

holds for all sufficiently large $n$. In fact, if $x>0$, then (2) follows by induction on $n$. If $x<-1$, then $U x=\frac{x}{x+1}>0$ which implies (2). For any $x \in(-1,0)$ there exists $m \in \mathbb{N}$ such that $-\frac{1}{m}<x<-\frac{1}{m+1}$; in this case we find $U^{m} x>0$, whence (2) holds for all sufficiently large $n$.

It follows from (2) that

$$
\delta_{j}^{(n)}(\alpha) \ll 1+\left(1+\frac{1}{n}\right)^{-2}<2\left(1-\frac{1}{n}\right)
$$

as $n \rightarrow \infty$. Thus

$$
\Sigma_{n} \ll 2\left(1-\frac{1}{n}\right) \Sigma_{n-1}
$$

and by induction

$$
\begin{aligned}
& 2^{1-n} \sum_{j=1}^{2^{n-1}} x_{j}^{(n)}(\alpha)-2^{1-n} \sum_{j=1}^{2^{n-1}} x_{j}^{(n)} \\
& \quad=2^{1-n} \Sigma_{n} \ll 2^{2-n}\left(1-\frac{1}{n}\right) \Sigma_{n-1} \ll 2 \prod_{m=2}^{n}\left(1-\frac{1}{m}\right)=\frac{2}{n}
\end{aligned}
$$


Now it follows from Theorem 2 that

$$
\lim _{n \rightarrow \infty} 2^{1-n} \sum_{j=1}^{2^{n-1}} x_{j}^{(n)}(\alpha)=\lim _{n \rightarrow \infty} 2^{1-n} \sum_{j=1}^{2^{n-1}} x_{j}^{(n)}=\frac{3}{2} .
$$

The theorem is proved.

\section{Acknowledgements}

The second and the third author are very grateful for the invitation to the Department of Mathematics at Clausthal University and the kind hospitality during their stay; they will never forget the hiking to the Brocken and the inspiring evenings at anno tobak.

\section{References}

[1] Alkauskas, G.; Steuding, J.: Statistical properties of the Calkin-Wilf tree: real and p-adic distribution. Preprint 2008, arXiv:0801.0054.

[2] Alperin, R.C.: Rationals and the modular group. Amer. Math. Monthly 106 (1999), 771-773.

[3] Bird, R.; Gibbons, J.; Lester, D.: Functional pearl: enumerating the rationals. J. Funct. Programming 16 (2006), 281-291.

[4] Brocot, A.: Calcul des rouages par approximation, nouvelle méthode. Revue Chrono-métrique 6 (1860), 186-194.

[5] Calkin, N.; Wilf, H.: Recounting the rationals. Amer. Math. Monthly 107 (2000), 360-363.

[6] Dilcher, K.; Stolarsky, K.B.: A polynomial analogue to the Stern sequence. J. Number Theory 3 (2007), 85-103.

[7] Eisenstein, G.: Über ein einfaches Mittel zur Auffindung der höheren Reciprocitätsgesetze und der mit ihnen zu verbindenden Ergänzungssätze. J. Reine Angew. Math. 39 (1850), 351-364.

[8] Graham, R.; Knuth, D.; Patashnik, O.: Concrete Mathematics: a foundation for computer science. Addison-Wesley, 1990.

[9] Hockman, M.: Continued fractions and the geometric decomposition of modular transformations. Quaestiones Math. 29 (2006), 1-20.

[10] Hofmann, S.; Schuster, G.; Steuding, J.: Euclid, Calkin \& Wilf - Playing with rationals. Elem. Math. 63 (2008), 109-117.

[11] Lagarias, J.C.; Tresser, C.: A walk along the branches of the extended Farey Tree. IBM J. Research Development 39 (1995), 283-294.

[12] Lehmer, D.H.: On Stern's diatomic series. Amer. Math. Monthly 36 (1929), 59-67.

[13] Newman, M.: Recounting the rationals, continued. Credited in: Amer. Math. Monthly 110 (2003), 642-643.

[14] Reznick, B.: Regularity properties of the Stern enumeration of the rationals. J. Integer Seq. 11 (2008), Article ID 08.4.1, 17 p. (electronic only).

[15] Series, C.: The geometry of Markoff numbers. Math. Intelligencer 7 (1985), 20-29.

[16] Serret, J.A.: Handbuch der höheren Algebra. Vol. 1, Teubner, 1868.

[17] Sloane, N.; Plouffe, S.: The On-line Encyclopedia of integer sequences. http: //www.research.att.com/ njas/sequences/ 
[18] Stern, M.A.: Über eine zahlentheoretische Funktion. J. Reine Angew. Math. 55 (1858), 193-220.

[19] Steuding, J.: Diophantine Analysis. CRC Press/Chapman Hall, 2005.

\section{Jürgen Sander}

Institut für Mathematik und Angewandte Informatik

Universität Hildesheim

Samelsonplatz 1

D-31141 Hildesheim, Germany

e-mail: sander@imai.uni-hildesheim.de

Jörn Steuding

Institut für Mathematik

Universität Würzburg

Am Hubland

D-97218 Würzburg, Germany

e-mail: steuding@mathematik.uni-wuerzburg.de

Rasa Steuding

Hochschule RheinMain Wiesbaden

Fachbereich Design Informatik Medien

Kurt-Schumacher-Ring 18

D-65197 Wiesbaden, Germany

e-mail: Rasa.Steuding@hs-rm.de 